\title{
Heat, cold and pressure induced denaturation of proteins
}

\author{
G. Panick, H. Herberhold, Z. Sun and R. Winter* \\ University of Dortmund, Department of Chemistry, Physical Chemistry I, Otto-Hahn Straße 6, D-44227 \\ Dortmund, Germany
}

\begin{abstract}
We studied the pressure-induced unfolding and refolding of monomeric proteins, such as SNase, $\alpha$-chymotrypsin and ubiquitin, by using synchrotron X-ray small-angle scattering and Fourier-transform infrared spectroscopy, which monitor changes in the tertiary and secondary structural properties of the proteins upon pressurization. Furthermore, by using the pressure-jump relaxation technique in combination with time-resolved X-ray diffraction and infrared spectroscopy, the kinetics of the unfolding/refolding of the proteins, was investigated. Significant differences in secondary structure and chain compactness in the folding/unfolding reactions of these proteins are observed. The results are compared with data obtained from other methods of denaturation, such as heat and pressure-assisted cold denaturation. The cold- and pressure-induced unfolding both yield a particularly unfolded state characterized by a persistent amount of secondary structure.
\end{abstract}

\section{Introduction}

In recent years we made extensive use of hydrostatic pressure as a physical parameter for studying the stability and energetics of lyotropic lipid mesophases, model biomembranes and proteins [1-5]. Combined with temperature dependent studies, the method revealed to be an interesting tool to explore the free energy landscape of these biomolecular systems. Recently we also focused on using pressure as a kinetic variable. Applying the pressure-jump relaxation technique in combination with time-resolved synchrotron X-ray diffraction, we studied the kinetics of various lipid phase transformations $[1,6]$. The technique can also be applied for studying transformations of other biomolecules, such as protein unfolding and refolding $[4,5,7]$. So far, most studies dealing with the kinetics of protein folding have been carried out at atmospheric pressure using temperature or chemical denaturants as experimental variables.

It has long been known that the application of hydrostatic pressure results in the disruption of native protein structures due to the decrease in the volume of the protein-solvent system upon unfolding, which arises - besides other factors - from the existence of solvent-excluded voids in the folded state. Pressure denaturation studies provide a fundamental thermodynamic parameter for protein unfolding, the volume change $\Delta V_{\mathrm{D}}^{\circ}$, in addition to an alternative method for perturbing the folded state, and thus extracting its stability, the standard Gibbs free energy change of denaturation, $\Delta G_{\mathrm{D}}^{\circ}$. The data obtained for the volume change, $\Delta V_{\mathrm{D}}^{\circ}$, allow to characterize the nature of the barrier to unfolding and the corresponding transition state. Moreover, in several cases pressure studies have been shown to present an important advantage due to the positive activation volume for folding, the result of which is to slow down folding substantially, in turn allowing for relatively straightforward measurements of structural order parameters that are difficult or even impossible to measure on much faster timescales [5,7].

${ }^{*}$ Corresponding author. Tel.: +49 231755 3900; Fax: +49 231755 3901; E-mail: winter@ steak.chemie.uni-dortmund.de. 
The cold unfolding of proteins is particulary interesting because a possible resemblance of the cold unfolded state of certain proteins, such as RNase, lysozyme and ubiquitin, with an early folding intermediate of these proteins at ambient conditions was suggested [8-10]. The nature of the cold unfolded state compared with the heat unfolded state is still a matter of controversy, however [9]. More recently, it was shown that high pressure and low temperature can also be applied to inactivate viruses [11].

In this paper we studied the cold unfolding of SNase and compare it with the pressure and heat unfolding as observed by Fourier-transform infrared (FT-IR) spectroscopy. The data are compared whith those for other small proteins, such as $\alpha$-chymotrypsin and ubiquitin. These proteins have traditionally served as models for protein folding because they are small and stable and have a well-known native structure.

\section{Experimental}

Details of the sample preparation and experimental techniques are discussed elsewhere [4,5,7]. Briefly, the high pressure FT-IR spectra were recorded with a Nicolet Magna 550 spectrometer equipped with a liquid nitrogen cooled $\mathrm{HgCdTe}$-detector. Heat unfolding was followed using a temperature cell with $\mathrm{CaF}_{2}$ windows separated by a $50 \mu \mathrm{m}$ teflon spacer, which was placed in the heating jacket controlled by an automatic temperature controller. Pressure unfolding was achieved using a diamond anvil cell (DAC) with type IIa diamonds, where the pressure was built up by means of a screw mechanism. Powdered $\alpha$-quartz was placed in the hole of the steel gasket of the DAC and changes in pressure were quantified by the shift of the quartz phonon band at $695 \mathrm{~cm}^{-1}$. The overlapping components of the amide $I^{\prime}$ band were narrowed by Fourier self-deconvolution. A resolution enhancement factor of 1.8 and a bandwidth of $15 \mathrm{~cm}^{-1}$ was used. The fractional intensities of the secondary structure elements were determined by fitting the self-deconvoluted amide $I^{\prime}$ band of the spectrum using Gaussian-Lorentzian line shape functions. The bands assigned to the different secondary structure elements are discussed in [4,12].

SNase was obtained as described in [4]. Ubiquitin and $\alpha$-chymotrypsin were purchased from Sigma Chemical Co. and used without further purification. The proteins were dissolved at a concentration of $5 \% \mathrm{w} / \mathrm{w}$ in $10 \mathrm{mM}$ TRIS-buffer (Sigma) with $99.9 \% \mathrm{D}_{2} \mathrm{O}$ (Sigma) at pD 5.5 or 7 for the high pressure experiments. For the temperature dependent experiments, we used a $10 \mathrm{mM}$ phosphate buffer. The $\mathrm{pD}$ value of the solution was adjusted using DCl. To ensure complete H/D exchange, the protein solutions were heated up to a temperature close to their denaturation temperature for one hour and then stored overnight at room temperature.

\section{Results and discussion}

\subsection{Pressure and temperature induced unfolding and denaturation}

Staphylococcal nuclease (SNase) is a small protein of about $17.5 \mathrm{kDa}$ containing 149 amino acids and no disulfide bonds. In the crystalline state, the protein contains $26.2 \%$ helices, $24.8 \% \beta$-sheets (barrel), 7.4\% extended chains, $24.8 \%$ turns and loops, and $8.7 \%$ unordered chains ( $8.1 \%$ are uncertain). Analysis of the high pressure SAXS data revealed [4,7] that over a pressure range from atmospheric pressure to approximately $3 \mathrm{kbar}$, the radius of gyration $R_{\mathrm{g}}$ of the protein increases from a value near $17 \AA$ for native SNase two-fold to a value near $35 \AA$. A large broadening of the pair-distribution function $p(r)$ was observed over the same range, indicating a transition from a globular to an ellipsoidal or dumbbell-like structure. For comparison, temperature-induced denaturation involves a further unfolding of the protein 

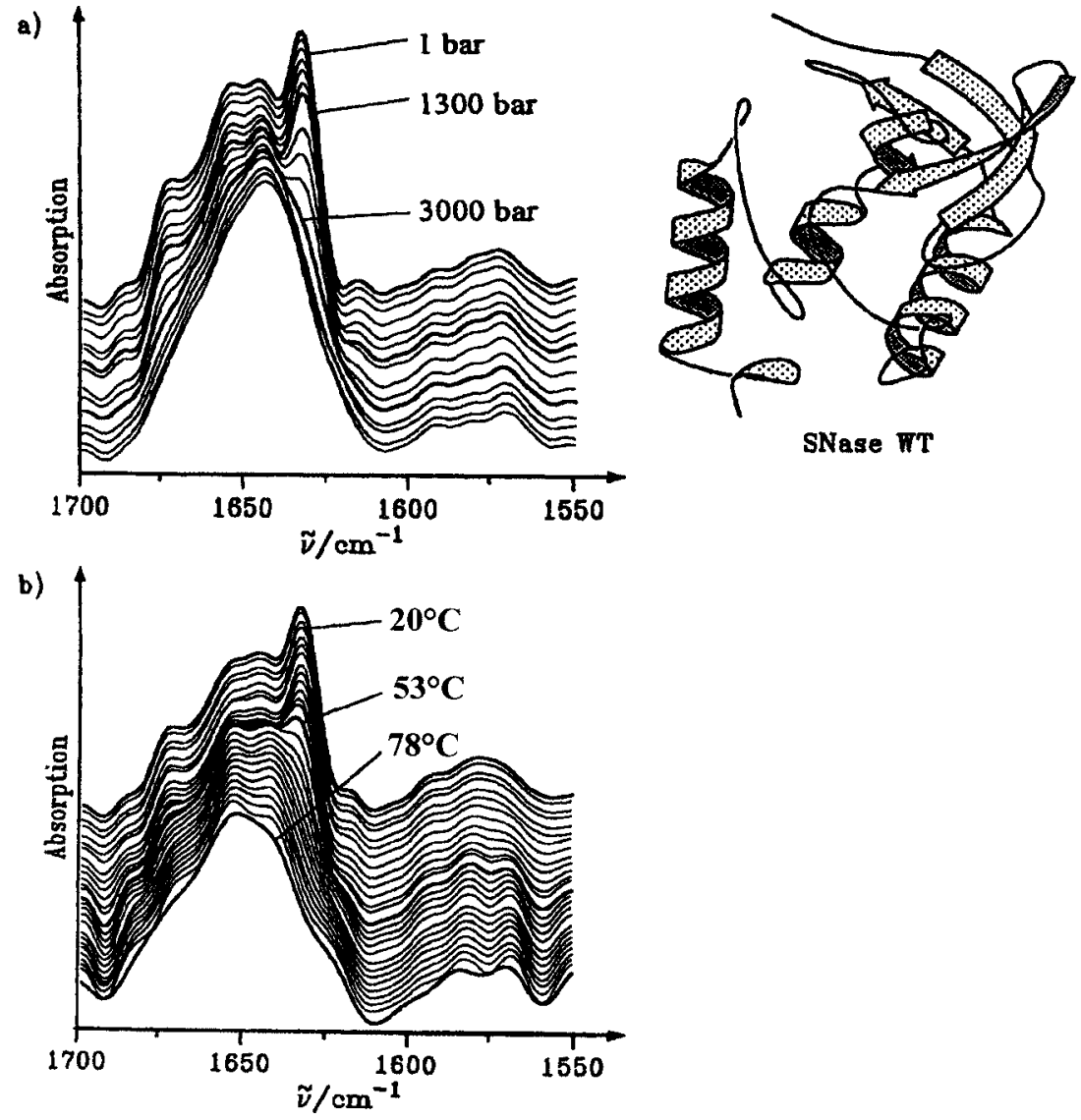

Fig. 1. Deconvoluted FT-IR spectra of SNase (5\% (w/w), pD 5.5) (a) at $25^{\circ} \mathrm{C}$ as a function of pressure and (b) as a function of temperature at ambient pressure (band assignment: $1611 \mathrm{~cm}^{-1}$ side chains, $1627 / 1673 \mathrm{~cm}^{-1} \beta$-sheets, $1651 \mathrm{~cm}^{-1} \alpha$-helices, $1641 / 1659 / 1666 \mathrm{~cm}^{-1}$ disordered structures/ turns). On the right-hand side a schematic drawing of the native state of wild-type SNase is shown.

molecule which is indicated by a larger $R_{\mathrm{g}}$-value of $45 \AA$ [4]. At temperatures above $\sim 40^{\circ} \mathrm{C}$, the structure of the pressure-denatured state ressembles that of the temperature-denatured state at ambient pressure, however.

Deconvolution of the FT-IR amide $I^{\prime}$ absorption band (Fig. 1a) reveals a pressure-induced denaturation process above about $2 \mathrm{kbar}$. The band at $1611 \mathrm{~cm}^{-1}$ can be assigned to side chains, the bands at $1627 \mathrm{~cm}^{-1}$ and $1673 \mathrm{~cm}^{-1}$ are due to $\beta$-sheets, the bands at $1641 \mathrm{~cm}^{-1}, 1659 \mathrm{~cm}^{-1}$ and $1666 \mathrm{~cm}^{-1}$ are associated with disordered and turn structures, and the band at $1651 \mathrm{~cm}^{-1}$ is assigned to $\alpha$-helices. As can be seen from Fig. 2, the denaturation process is evidenced by an increase in disordered and turn structures and a drastic decrease in the content of $\beta$-sheets and $\alpha$-helices. The pressure-induced denatured state above $3 \mathrm{kbar}$ retains nonetheless some degree of $\beta$-like secondary structure and the molecules cannot be described as a fully extended random polypeptide coil, which is in accord with the SAXS results (see above).

In order to determine the standard Gibbs free energy change $\Delta G_{\mathrm{D}}^{\circ}$ and the volume change $\Delta V_{\mathrm{D}}^{\circ}=$ $\mathrm{d} \Delta G_{\mathrm{D}}^{\circ} / \mathrm{d} p$ of a two-state pressure-induced unfolding process, the equilibrium profiles obtained from the 
FT-IR spectra were analysed according to the relation

$$
\Delta G_{\mathrm{D}}^{\circ}(p)=-R T \ln K_{\mathrm{eq}}(p)=-R T \ln \left(\left(I_{\mathrm{F}}-I_{\mathrm{p}}\right) /\left(I_{\mathrm{p}}-I_{\mathrm{D}}\right)\right)
$$

using the data points at each pressure, $I_{\mathrm{p}}$, and the asymptotic values of the fractional intensity due to particular secondary structural elements for the folded and unfolded (denatured) states, $I_{\mathrm{F}}$ and $I_{\mathrm{D}}$, respectively. Assuming the pressure-induced unfolding transition of SNase to occur essentially as a two-state process, analysis of the FT-IR pressure profiles yields a standard Gibbs free energy change for unfolding of $\Delta G_{\mathrm{D}}^{\circ}=17 \pm 4 \mathrm{~kJ} / \mathrm{mol}$ and a volume change for unfolding of $\Delta V_{\mathrm{D}}^{\circ}=-80 \pm 20 \mathrm{ml} / \mathrm{mol}$ at ambient temperature and pressure.

Figure $1 \mathrm{~b}$ exhibits the deconvoluted temperature dependent spectra of SNase at ambient pressure. A reversible thermal transition takes place between $40^{\circ} \mathrm{C}$ and $60^{\circ} \mathrm{C}$. The temperature-induced decrease of band intensities of $\beta$-sheets and $\alpha$-helices (Fig. 3) is accompanied by an increase in band intensities of

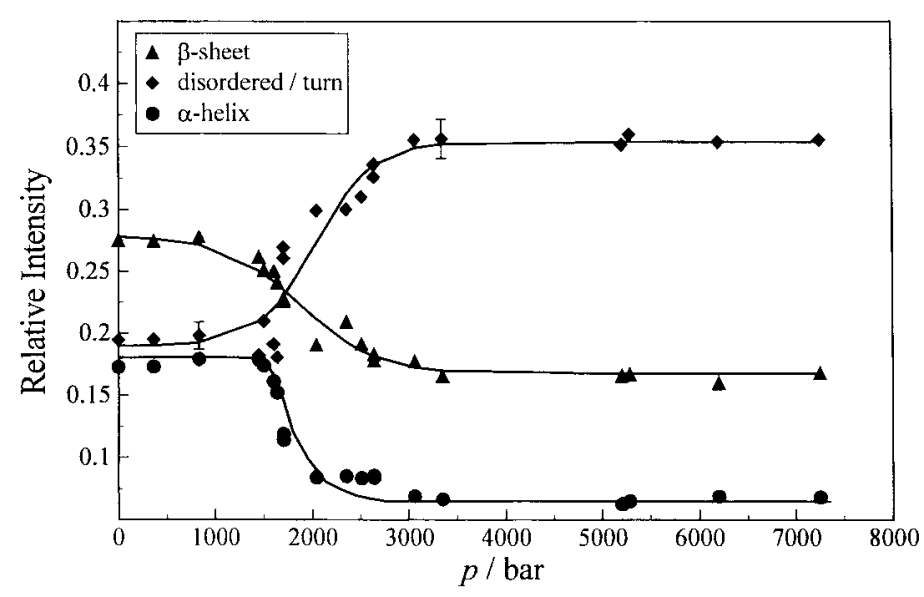

Fig. 2. Pressure effect on the areas of the bands associated with $\beta$-sheets, disordered/turn structures and $\alpha$-helices of SNase at $\mathrm{pD} 5.5$ and $25^{\circ} \mathrm{C}$.

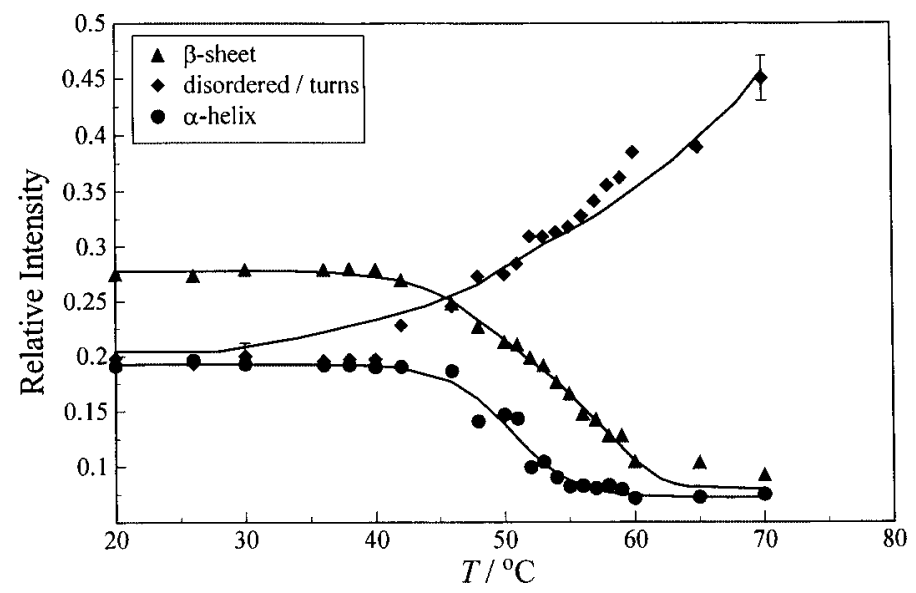

Fig. 3. Temperature effect on the areas of the bands associated with $\beta$-sheets, disordered/turn structures and $\alpha$-helices of SNase at pD 5.5 and $25^{\circ} \mathrm{C}$. 
disordered and turn structures. Comparison with the pressure-induced denaturation data for the fractional intensities (Fig. 2) reveals that in contrast to the effects of pressure, the bands due to $\beta$-sheet structures are completely disrupted by high temperature. Xie et al. (1996) also conclude that at $70^{\circ} \mathrm{C}$ defined structures, such as $\alpha$-helices and $\beta$-sheets, are destroyed and only some uncertain non-random structures remain.

\subsection{Cold induced unfolding}

The usually observed positive denaturational increment of heat capacity of proteins means that the enthalpy of protein denaturation is a temperature dependent function [14]. Hence, one can expect that the enthalpy of denaturation can, in principle, become zero and then even invert its sign at some low enough temperature, changing from the factor stabilizing the native protein structure into a factor destabilizing its structure. Therefore, one can imagine that protein denaturation can occur not only upon heating but also upon cooling. In contrast to heat denaturation, cold denaturation should then proceed with a release of heat, i.e., a decrease of enthalpy. Unfortunately, for most proteins the low temperature part of the heat capacity function that is connected with cold denaturation takes place at too low temperatures to be traced to its completeness even in the supercooled solution. To be able to study the system at temperatures below $0^{\circ} \mathrm{C}$, one may apply moderate pressures which allow studies of aqueous solutions down to temperatures of about $-15^{\circ} \mathrm{C}$. By these means the pressure-assisted cold denatured state of many proteins is accessible.

Recent measurements of the heat-induced unfolding of SNase showed [15] that it is accompanied by a large increase in heat capacity of $8.3 \mathrm{~kJ} /(\mathrm{mol} \cdot \mathrm{K})$, which indicates that cold denaturation of this potein might be observable under experimentally realizable conditions. By using the experimentally determined values of $\Delta C_{\mathrm{p}}, T_{\mathrm{m}}$ and $\Delta H$ of unfolding, we can determine by Eq. (2) the standard Gibbs free energy difference between the denatured and native state for any chosen temperature:

$$
\Delta G_{\mathrm{D}}^{\circ}(T)=\Delta H_{\mathrm{D}}^{\circ}\left(T_{\mathrm{m}}\right)\left(1-\frac{T}{T_{\mathrm{m}}}\right)+\Delta C_{\mathrm{p}}\left(T-T_{\mathrm{m}}\right)+\Delta C_{\mathrm{p}} T \ln \left(\frac{T_{\mathrm{m}}}{T}\right)
$$

( $T_{\mathrm{m}}$ midpoint of unfolding transition at ambient pressure) [15]. As seen from Fig. 4, it is a function with an extremum and crosses the zero level at two different temperatures, $T_{\mathrm{m}}$, and $T_{\mathrm{c}}$. According to the figure, heat denaturation occurs at $48^{\circ} \mathrm{C}$ and cold denaturation would occur around $-13.6^{\circ} \mathrm{C}$ at ambient pressure. As the stability of the native structure of the protein, $\Delta G_{\mathrm{D}}^{\circ}$, is zero at these temperatures, it is clear that the first of these temperatures is the temperature of the heat denaturation of the protein molecule

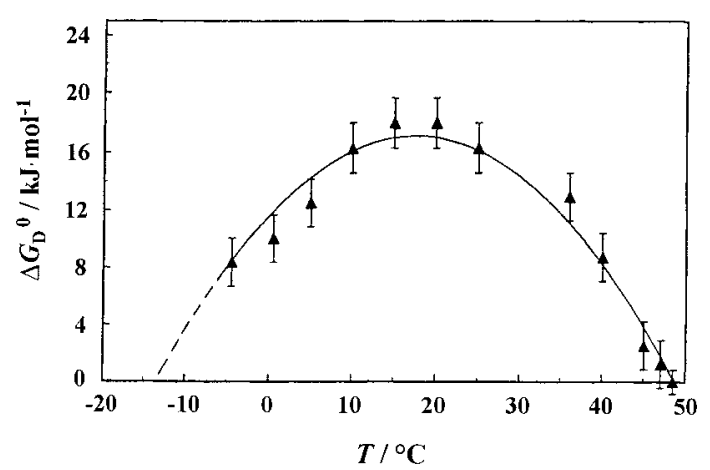

Fig. 4. Temperature dependence of the equilibrium standard Gibbs free energy of unfolding, $\Delta G_{\mathrm{D}}^{\circ}$, at ambient pressure. 


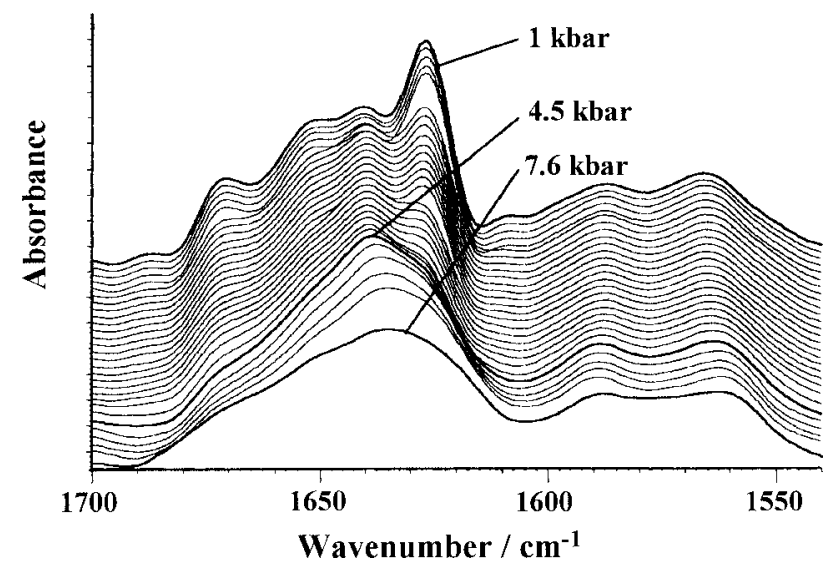

Fig. 5. Deconvoluted FT-IR spectra of SNase (5\% (w/w), pD 5.5) at $-4.5^{\circ} \mathrm{C}$ as a function of pressure (band assignment: $1611 \mathrm{~cm}^{-1}$ side chains, $1627 / 1673 \mathrm{~cm}^{-1} \beta$-sheets, $1651 \mathrm{~cm}^{-1} \alpha$-helices, $1641 / 1659 / 1666 \mathrm{~cm}^{-1}$ disordered structures/turns).

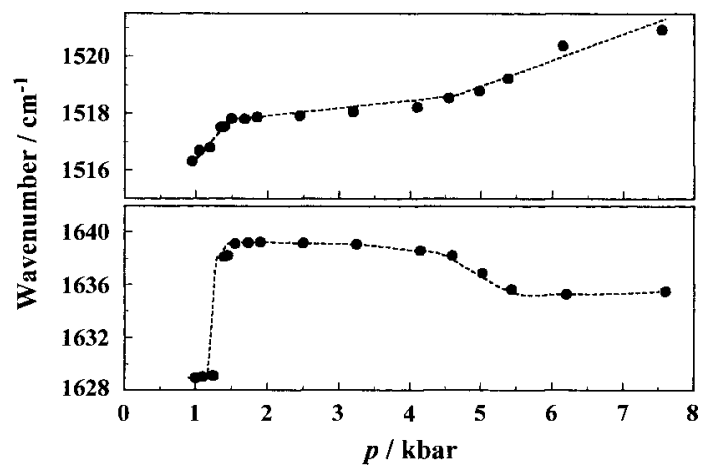

Fig. 6. Pressure dependence of the position of the tyrosine band (top) and the maximum of the amide $I^{\prime}$ band (bottom) of SNase at $-4.5^{\circ} \mathrm{C}(\mathrm{pD} 5.5)$.

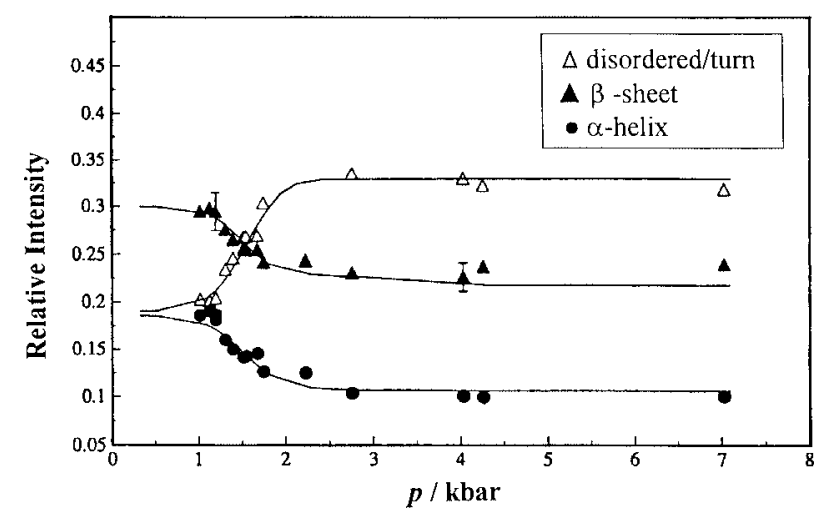

Fig. 7. Pressure effect on the areas of the bands associated with $\beta$-sheets, disordered/turn structures and $\alpha$-helices of SNase at pD 5.5 and $-4.5^{\circ} \mathrm{C}$. 


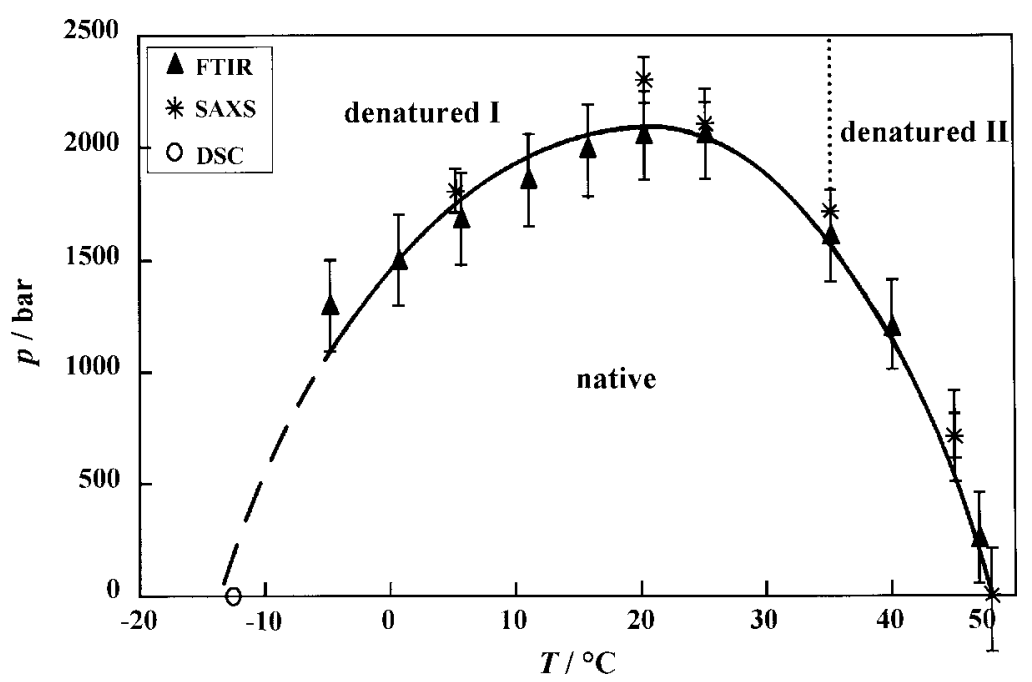

Fig. 8. $p, T-$ stability diagram of SNase $\mathrm{WT}$ at $\mathrm{pD} 5.5$.

$\left(T_{\mathrm{m}}\right)$ and the second one is that of its cold denaturation $\left(T_{\mathrm{c}}\right)$. The latter one is in good agreement with calorimetric data [14].

Figure 5 shows the self deconvoluted FT-IR equilibrium spectra of SNase at $-4.5^{\circ} \mathrm{C}$ up to pressures of 7.5 kbar. Figure 6 exhibits the pressure dependent maximum wavenumbers of the amide $I^{\prime}$ and tyrosine absorption band. Clearly, a transition pressure from the native to a denatured state occurs around $1.4 \mathrm{kbar}$. As the features of the tyrosine and amide $I^{\prime}$ spectra occur concomitantly, we may assume that the changes of the secondary structure elements and tertiary contacts around the tyrosine residues occur at the same pressure, pointing to a two-state kind of transition. Above about $4.5 \mathrm{kbar}$, the maxima of the bands shift which is due the pressure-induced formation of ice V. The content of secondary structure elements is not influenced by the freezing the bulk liquid, however (Fig. 7).

The pressure midpoints at several temperatures obtained from the FT-IR and SAXS profiles are plotted as a stability diagram of the protein in Fig. 8. It exhibits the elliptic-like curvature which is typical of heat and cold denaturation of many monomeric proteins. As the SAXS and FT-IR data show that the heat and pressure induced denatured states have different global structures and different secondary structure elements, the denatured states have tentatively been classified as denatured I and denatured II, respectively. The maximum pressure stability of the protein appears to be at a temperature close to $20^{\circ} \mathrm{C}$.

In Fig. 9, the stability diagram for SNase is compared to that of two other monomeric proteins, ubiquitin and $\alpha$-chymotrypsin. As can be clearly seen, the pressure stability of SNase is rather low. In fact, most monomeric proteins seem to unfold at pressures of about 4-8 kbar at room temperature [2,9]. Contrary to most proteins studied so far, part of the secondary structure of ubiquitin rearranges at high pressure forming new ordered structures [10].

\subsection{Kinetics of unfolding and refolding}

By crossing the phase boundary applying a pressure-jump, the folding and refolding kinetics can be studied. After a $p$-jump from 1 bar to $2.4 \mathrm{kbar}$ at $20^{\circ} \mathrm{C}$, the $R_{\mathrm{g}}$ increases in a first-order manner from 17 to $22.4 \AA$ over a timescale of approximately $30 \mathrm{~min}$ [4,7] (Fig. 10). The increase in $R_{\mathrm{g}}$ value is caused by the formation of an ellipsoidal structure as indicated by the corresponding pair-distance distribution function. Pressure-jump FT-IR studies [4] reveal that the reversible first-order changes in $\beta$-sheet, $\alpha$-helical 


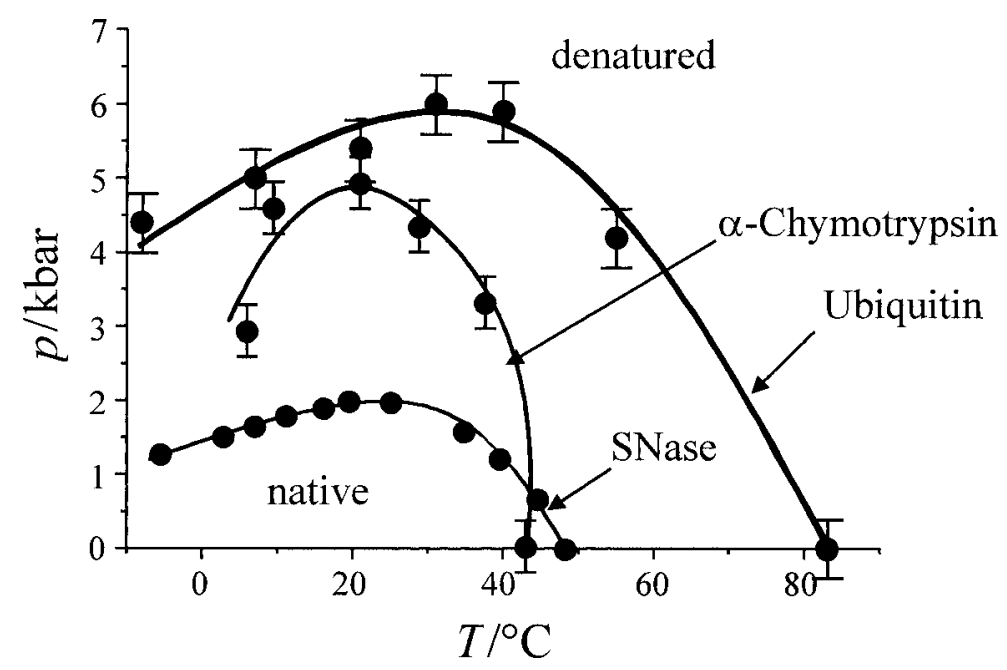

Fig. 9. Comparison of the $p, T$ - stability diagram of several monomeric proteins (pD 5.5 for SNase, pD 7.0 for ubiquitin and $\alpha$-chymotrypsin).

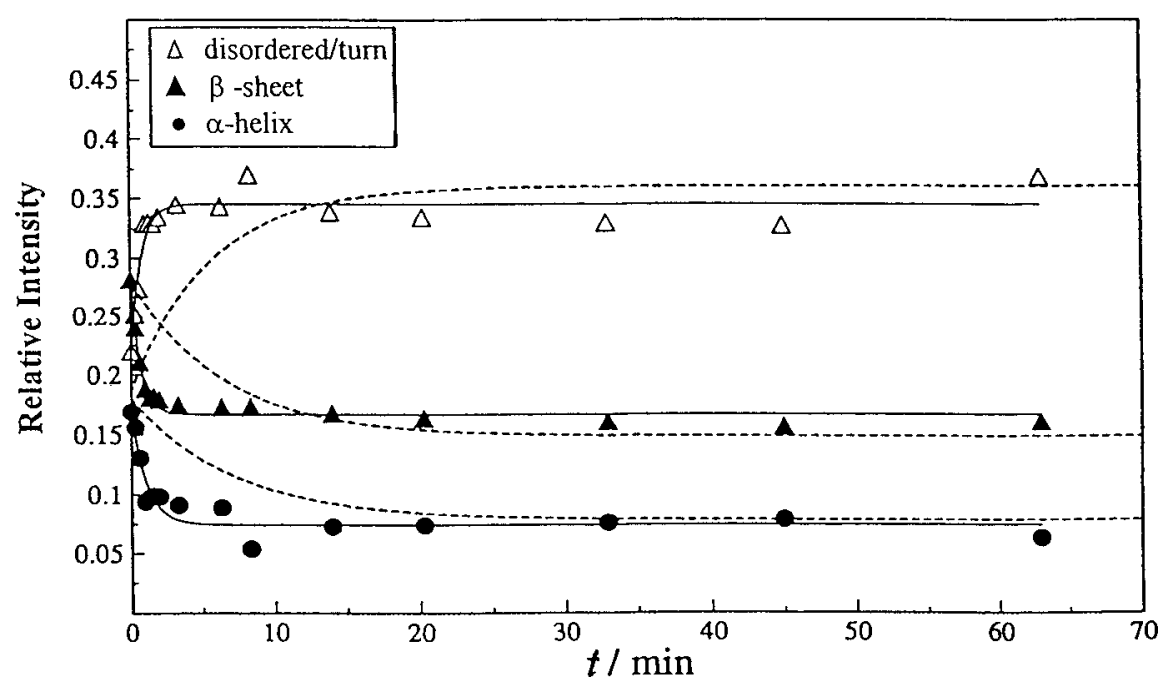

Fig. 10. Time-dependent fractional intensities of secondary structure elements of SNase after a $p$-jump from 1 to $3 \mathrm{kbar}$ at $T=20^{\circ} \mathrm{C}$ (dashed line) and $T=40^{\circ} \mathrm{C}$ (data points).

and random structure occur on the same slow timescale as that observed for the scattering curves. The $p$-jump FT-IR, SAXS and fluorescence emission [16] relaxation profiles can be fitted to a single exponential decay kinetics and their analysis yields values for the rate constants and activation volumes. The characteristic relaxation time $\tau$ at $20^{\circ} \mathrm{C}$ for unfolding is $6.2 \pm 0.7 \mathrm{~min}$ and that for refolding is much smaller $(1.2 \pm 0.5 \mathrm{~min})$. Figure 11 exhibits the relaxation time for unfolding as a function of the final pressure reached. The data show that above $3 \mathrm{kbar}$ the relaxation time is independent of the pressurejump amplitude. An increase of temperature leads to an increase in rate constants, e.g., $\tau$ for unfolding at $40^{\circ} \mathrm{C}$ is $2.5 \pm 0.4 \mathrm{~min}$ (Fig. 10). We find that the activation volume for folding is large and positive $(57 \pm 4 \mathrm{ml} / \mathrm{mol})$ and that for unfolding seems to be small and negative $(-23 \pm 3 \mathrm{ml} / \mathrm{mol})[4,5,7]$. An 


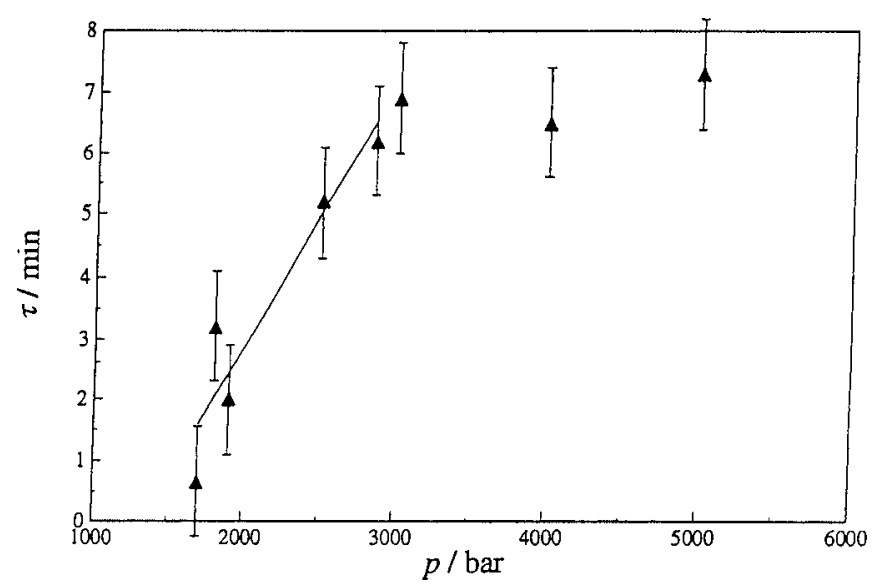

Fig. 11. Relaxation time for unfolding after pressure jumps from $1 \mathrm{kbar}$ (native state) to pressures above $1.7 \mathrm{kbar}\left(\mathrm{T}=20^{\circ} \mathrm{C}\right.$, pD 5.5).

increase of pressure would thus lead to a decrease in the rate constant of folding. The volume of the protein solvent system in the transition state is thus significantly larger than in the unfolded state and somewhat smaller than in the folded state, so that the transition state lies closer to the folded than to the unfolded state in terms of system volumes.

To summarize, the present pressure-jump SAXS and FT-IR data, along with previous fluorescence pressure-jump results, provide strong evidence for a two-state folding/unfolding model for SNase under pressure, in that secondary structure, chain collapse and tertiary structure all exhibit relaxation profiles on similar timescales. Thus, changes in these three order parameters appear to depend upon the same rate-limiting steps for unfolding and folding. The positive activation volume for folding, which is responsible for the large increase in the relaxation time of folding with pressure (allowing us to observe these processes without resorting to ultrafast methods), is consistent with a model in which collapse with concomitant desolvation constitutes the rate limiting step in folding. We also showed for SNase that the cold, pressure and heat unfolding do not follow the same pathway but rather probe different pathways of a rugged folding funnel. Generally, all experimental evidence indicates that the pressure-denatured and cold-denatured states of proteins have more secondary structure than the temperature-denatured states. Our data also show that the pressure stability of monomeric proteins may be significantly different. As in the case of ubiquitin, part of the protein may even reorganize at high pressure and new secondary structure elements may be formed.

\section{Acknowledgments}

This work was supported by the Deutsche Forschungsgemeinschaft (DFG) and the Fonds der Chemischen Industrie.

\section{References}

[1] R. Winter, Biochim, Biophys. Acta 1595 (2002), 160-184.

[2] R. Winter and J. Jonas, eds, High Pressure Molecular Science, NATO ASI E, Vol. 358, Kluwer Academic Publishers, Dordrecht, The Netherlands, 1999. 
[3] R. Winter, J. Erbes, C. Czeslik and A. Gabke, J. Phys.: Condens. Matter 10 (1998), 11499-11518.

[4] G. Panick, R. Malessa, R. Winter, G. Rapp, K.J. Frye and C. Royer, J. Mol. Biol. 275 (1998), 389-402.

[5] G. Panick, G.J.A. Vidugiris, R. Malessa, G. Rapp, R. Winter and C. Royer, Biochemistry 38 (1999), 4157-4164.

[6] J. Erbes, A. Gabke, G. Rapp and R. Winter, Phys. Chem. Chem. Phys. 2 (2000), 151-162.

[7] J. Woenckhaus, R. Köhling, P. Thiyagarajan, K. Littrell, S. Seifert, C.A. Royer and R. Winter, Biophys. J. 80 (2001), $1518-1523$.

[8] D.P. Nash and J. Jonas, Biochem. Biophys. Res. Commun. 238 (1997), 289-291.

[9] F. Meersman, L. Smeller and K. Heremans, Biophys. J. 82 (2002), 2635-2644.

[10] H. Herberhold and R. Winter, Biochemistry 41 (2002), 2396-2401.

[11] A.C.D. Oliveira, D. Ishimaru, R.B. Goncalves, T.J. Smith, P. Masson, D. Sa-Carvalho and J.L. Silva, Biophys. J. 76 (1999), $1270-1279$.

[12] D.M. Byler and H. Susi, Biopolymers 25 (1986), 469-487.

[13] L. Xie, G.-Z. Jing and J.-M. Zhou, Arch. Biochem. Biophys. 328 (1996), 122-128.

[14] Y.V. Griko, P.L. Privalov, J.M. Sturtevant and S.Y. Venyaminov, Proc. Natl. Acad. Sci. 85 (1988), 3343-3347.

[15] H. Seemann, R. Winter and C.A. Royer, J. Mol. Biol. 307 (2001), 1091-1102.

[16] G.J.A. Vidugiris, J.L. Markley and C.A. Royer, Biochemistry 34 (1995), 4909-4912. 


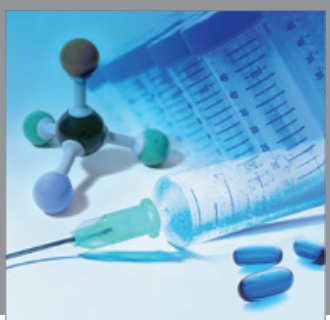

International Journal of

Medicinal Chemistry

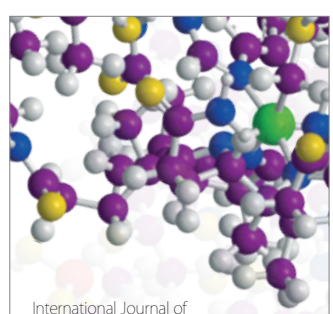

Carbohydrate Chemistry

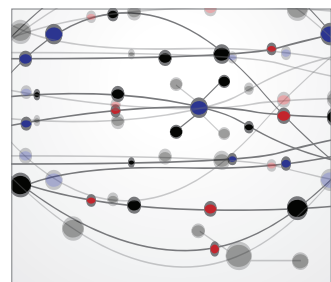

The Scientific World Journal
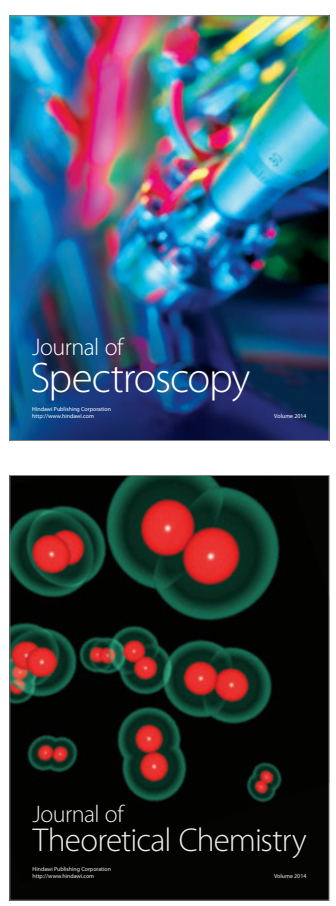
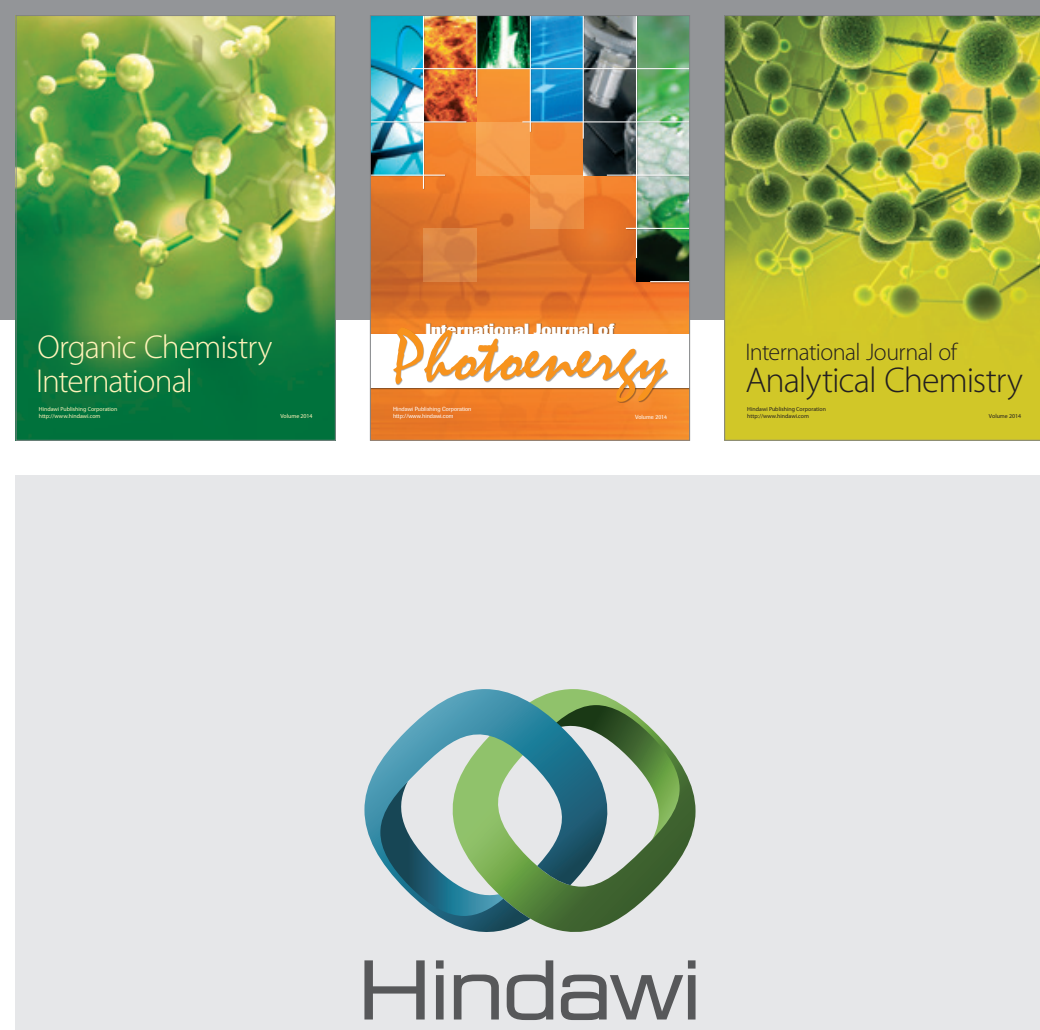

Submit your manuscripts at

http://www.hindawi.com
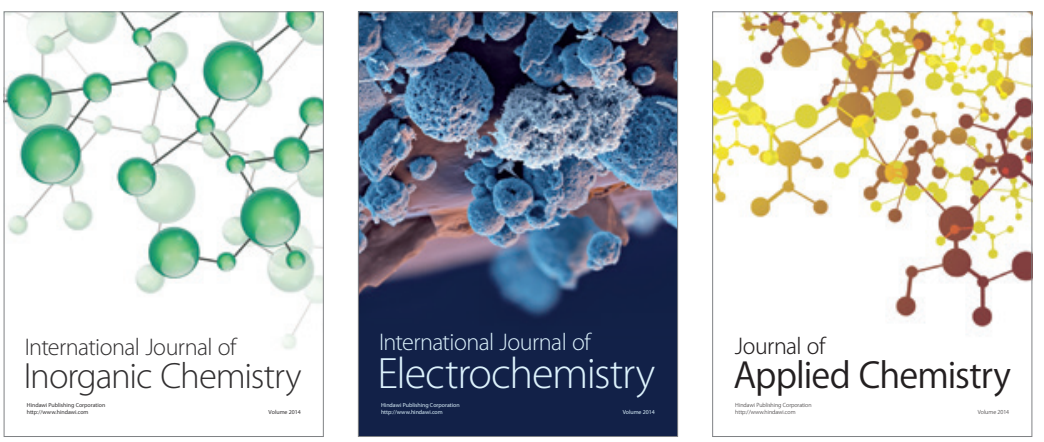

Journal of

Applied Chemistry
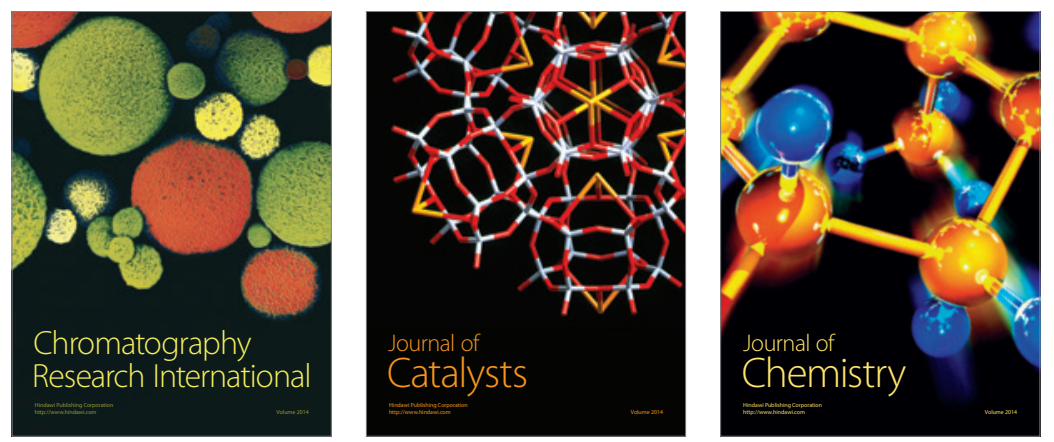
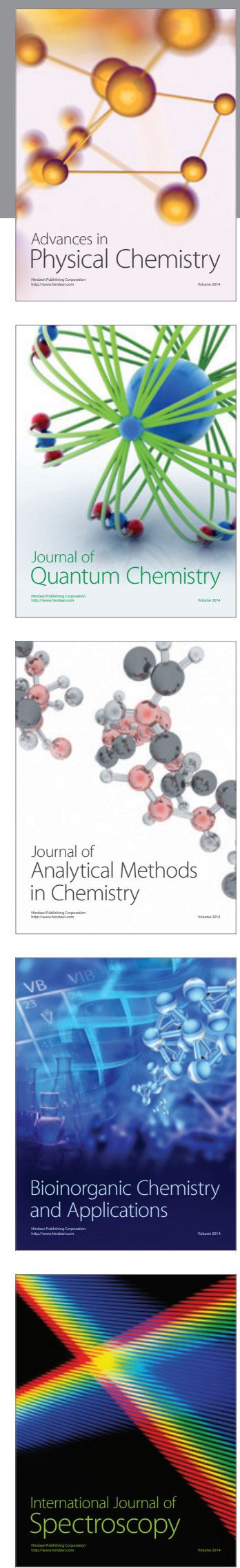